\title{
Vaccine effectiveness against SARS-CoV-2 transmission and infections among household and other close contacts of confirmed cases, the Netherlands, February to May 2021
}

Brechje de Gier ${ }^{1}$, Stijn Andeweg ${ }^{1}$, Rosa Joosten ${ }^{1}$, Ronald ter Schegget ${ }^{2}$, Naomi Smorenburg ${ }^{1}$, Jan van de Kassteele ${ }^{1}$, RIVM COVID-19 surveillance and epidemiology team ${ }^{1,3}$, Susan JM Hahné ${ }^{1}$, Susan van den Hof ${ }^{1}$, Hester E de Melker ${ }^{1}$, Mirjam J Knol ${ }^{1}$

1. Center for Infectious Disease Control, National Institute for Public Health and the Environment (RIVM), Bilthoven, the Netherlands

2. GGD Brabant Zuidoost, Eindhoven, the Netherlands

3. The members of the group are listed under Investigators

Correspondence: Brechje de Gier (brechje.de.gier@rivm.nl)

Investigators: The investigators are listed at the end of the article.

Citation style for this article:

de Gier Brechje, Andeweg Stijn, Joosten Rosa, ter Schegget Ronald, Smorenburg Naomi, van de Kassteele Jan, RIVM COVID-19 surveillance and epidemiology team 1 , Hahné Susan JM, van den Hof Susan, de Melker Hester E, Knol Mirjam J. Vaccine effectiveness against SARS-CoV-2 transmission and infections among household and other close contacts of confirmed cases, the Netherlands, February to May 2021. Euro Surveill. 2021;26(31):pii=2100640. https://doi.org/10.2807/1560-7917.

ES.2021.26.31.2100640

Several studies report high effectiveness of COVID19 vaccines against SARS-CoV-2 infection and severe disease, however an important knowledge gap is the vaccine effectiveness against transmission (VET). We present estimates of the VET to household and other close contacts in the Netherlands, from February to May 2021, using contact monitoring data. The secondary attack rate among household contacts was lower for fully vaccinated than unvaccinated index cases ( $11 \%$ vs $31 \%$ ), with an adjusted VET of $71 \%$ ( $95 \%$ confidence interval: $63-77$ ).

An important question when making prognoses of the pandemic in the near future and of the need on nonpharmaceutical control measures is to what extent the vaccines reduce the likelihood of transmission from infected vaccinees. Based on routine contact monitoring data, we here estimate the vaccine effectiveness against transmission (VET) and the vaccine effectiveness against infection (VE) among household and other close contacts of confirmed cases of severe acute respiratory syndrome coronavirus 2 (SARS-CoV-2) infection in the Netherlands, between 1 February and 27 May 2021. The Alpha variant (Phylogenetic Assignment of Named Global Outbreak (Pango) lineage designation B.1.1.7) was the dominant variant in the area at that time.

\section{Source and contact tracing}

In the Netherlands, people are encouraged to undergo SARS-CoV-2 testing free of charge when experiencing symptoms or after contact with a confirmed case [1].
Infections confirmed by PCR, loop mediated isothermal amplification (LAMP) or antigen test are notified to the regional Municipal Health Services (MHS), who perform source and contact tracing and contact monitoring [2]. During our study period, household members and other close contacts of confirmed cases needed to quarantine for 10 days post exposure. All close contacts of a confirmed case were encouraged to get tested as soon as possible after exposure. In addition, a test was recommended on the $5^{\text {th }}$ day after last exposure. If negative, contacts could end quarantine. We obtained a pseudonymised minimal contact monitoring dataset from all MHS. Additional data on index cases and contacts who tested positive, including the vaccine received and date of symptom onset, was extracted from the national infectious disease notification registry. Of note, a contact becomes an index case when testing positive, so our study could include some persons both as contact and as index case.

\section{Vaccination status}

The time since vaccination of the index case was based on the number of days between vaccination and a date used for statistics (DUFS), which was either the reported date of symptom onset or, if that was unknown, the date of positive test result minus 2 days. For vaccinated contacts, time since vaccination was calculated as the number of days between vaccination and the date of first exposure of the contact to the index case within the infectious period of the index, which is defined during source and contact tracing as 2 days before symptom onset or 2 days before test. Partly vaccinated 
Characteristics of COVID-19 index cases (18 years and older), by vaccination status of the index and characteristics of contacts, and by vaccination status of the contact, the Netherlands, 1 February-27 May 2021 ( $\mathrm{n}=113,582$ index cases, $\mathrm{n}=253,168$ contacts)

\begin{tabular}{|c|c|c|c|c|c|c|c|c|c|c|c|c|c|}
\hline & & \multicolumn{6}{|c|}{ Vaccination status index } & \multicolumn{6}{|c|}{ Vaccination status contacts } \\
\hline & & \multicolumn{2}{|c|}{ Unvaccinated } & \multicolumn{2}{|c|}{$\begin{array}{c}\text { Partly } \\
\text { vaccinated }\end{array}$} & \multicolumn{2}{|c|}{$\begin{array}{c}\text { Fully } \\
\text { vaccinated }\end{array}$} & \multicolumn{2}{|c|}{ Unvaccinated } & \multicolumn{2}{|c|}{$\begin{array}{c}\text { Partly } \\
\text { vaccinated }\end{array}$} & \multicolumn{2}{|c|}{$\begin{array}{c}\text { Fully } \\
\text { vaccinated }\end{array}$} \\
\hline & & $\mathrm{n}$ & $\%^{\mathrm{a}}$ & $\mathrm{n}$ & $\%^{a}$ & $\mathrm{n}$ & $\%^{a}$ & $n$ & $\%^{\mathrm{a}}$ & $n$ & $\%^{a}$ & $n$ & $\%^{\mathrm{a}}$ \\
\hline \multicolumn{2}{|l|}{ Total } & \multicolumn{2}{|c|}{110,872} & \multicolumn{2}{|c|}{2,088} & \multicolumn{2}{|c|}{622} & \multicolumn{2}{|c|}{243,360} & \multicolumn{2}{|c|}{4,411} & \multicolumn{2}{|c|}{5,397} \\
\hline \multirow{2}{*}{$\begin{array}{l}\text { Number of contacts } \\
\text { by type }\end{array}$} & Household & 139,802 & 56 & 2,032 & 50 & 706 & 55 & 138,095 & 57 & 1,917 & 43 & 2,528 & 47 \\
\hline & Other close contacts & 108,041 & 44 & 2,004 & 50 & 583 & 45 & 105,265 & 43 & 2,494 & 57 & 2,869 & 53 \\
\hline \multirow{3}{*}{ Sex } & Female & 56,554 & 51 & 1,325 & 63 & 472 & 76 & 121,183 & 50 & 2,689 & 61 & 4,139 & 77 \\
\hline & Male & 54,318 & 49 & 763 & 37 & 150 & 24 & 120,473 & 50 & 1,684 & 38 & 1,216 & 23 \\
\hline & Unknown/ other & 0 & 0 & 0 & 0 & 0 & 0 & 1,704 & 1 & 38 & 1 & 42 & 1 \\
\hline \multirow{7}{*}{ Age (years) } & $0-11$ & 0 & 0 & 0 & 0 & 0 & 0 & 42,119 & 17 & 0 & 0 & 0 & 0 \\
\hline & $12-17$ & 0 & 0 & 0 & 0 & 0 & 0 & 19,770 & 8 & 0 & 0 & 0 & 0 \\
\hline & $18-29$ & 31,736 & 29 & 209 & 10 & 122 & 20 & 57,264 & 24 & 437 & 10 & 961 & 18 \\
\hline & $30-49$ & 42,142 & 38 & 347 & 17 & 179 & 29 & 54,591 & 22 & 562 & 13 & 1,102 & 20 \\
\hline & $50-74$ & 34,383 & 31 & 1,155 & 55 & 194 & 31 & 58,688 & 24 & 2,688 & 61 & 2,280 & 42 \\
\hline & $\geq 75$ & 2,611 & 2 & 377 & 18 & 127 & 20 & 4,321 & 2 & 724 & 16 & 1,054 & 20 \\
\hline & Unknown & 0 & 0 & 0 & 0 & 0 & 0 & 6,607 & 3 & 0 & 0 & 0 & 0 \\
\hline \multirow{5}{*}{ Vaccine received } & Vaxzevria & \multicolumn{2}{|l|}{ NA } & 1,144 & 55 & 35 & 6 & \multicolumn{2}{|c|}{ NA } & 2,127 & 48 & 407 & 8 \\
\hline & Comirnaty & \multicolumn{2}{|l|}{ NA } & 890 & 43 & 530 & 85 & \multicolumn{2}{|c|}{ NA } & 1,235 & 28 & 3,312 & 61 \\
\hline & Janssen & \multicolumn{2}{|l|}{ NA } & 0 & 0 & 21 & 3 & \multicolumn{2}{|c|}{ NA } & 0 & 0 & 83 & 2 \\
\hline & Spikevax & \multicolumn{2}{|l|}{ NA } & 54 & 3 & 36 & 6 & \multicolumn{2}{|c|}{ NA } & 86 & 2 & 247 & 5 \\
\hline & Unknown & \multicolumn{2}{|l|}{ NA } & 0 & 0 & 0 & 0 & NA & & 963 & 22 & 1,348 & 25 \\
\hline & Couple with children & 12,782 & 15 & 117 & 8 & 61 & 14 & 34,603 & 25 & 126 & 7 & 286 & 11 \\
\hline Household & $\begin{array}{l}\text { Couple without } \\
\text { children }\end{array}$ & 33,096 & 40 & 809 & 58 & 181 & 43 & 32,264 & 23 & 908 & 47 & 914 & 36 \\
\hline composition $^{b}$ & $\begin{array}{l}\text { Household with > two } \\
\text { adults }\end{array}$ & 21,459 & 26 & 272 & 19 & 112 & 27 & 55,408 & 40 & 648 & 34 & 1,062 & 42 \\
\hline & Other & 16,056 & 19 & 197 & 14 & 68 & 16 & 15,820 & 11 & 235 & 12 & 266 & 11 \\
\hline & Feb & 29,953 & 27 & 196 & 9 & 43 & 7 & 62,213 & 26 & 182 & 4 & 374 & 7 \\
\hline Month of notification & Mar & 38,573 & 35 & 435 & 21 & 143 & 23 & 88,116 & 36 & 738 & 17 & 1,571 & 29 \\
\hline case & Apr & 20,648 & 19 & 448 & 21 & 151 & 24 & 45,977 & 19 & 919 & 21 & 1,252 & 23 \\
\hline & May & 21,698 & 20 & 1,009 & 48 & 285 & 46 & 47,054 & 19 & 2,572 & 58 & 2,200 & 41 \\
\hline
\end{tabular}

COVID-19: coronavirus disease; NA: not applicable.

${ }^{a}$ Column percentage.

${ }^{b}$ Only presented for index cases with household contacts $(n=85,210)$ and for household contacts $(n=142,540)$.

was defined as having received the first dose of a twodose coronavirus disease (COVID-19) vaccine, with a time since vaccination of at least 14 days. Fully vaccinated was defined as having completed a two-dose schedule with a time since vaccination of at least 7 days, or the one-dose Janssen (Ad26.COV2-S (recombinant), Janssen-Cilag International NV, Beerse, Belgium) schedule with a time since vaccination of at least 14 days. We included only index cases aged 18 years or older because children were not eligible for vaccination at the time. Contacts aged $0-17$ years were included in the VET analyses, but not in the VE analyses. In order to exclude co-primary cases, the household contacts of an index were excluded if the most likely setting of infection of the index was 'at home' according to the source tracing interview (excluding 44,676 contacts $(15 \%))$. Further, only SARS-CoV-2-positive contacts with a DUFS within 1 to 14 days after the DUFS of the index case were included in the analyses, to reduce misclassification of indexes and secondary cases.

\section{Index cases and contacts}

The final dataset contained 253,168 contacts of 113,582 index cases $(5,394$ persons in our study were both contact and index case). Of the index cases, $622(0.5 \%)$ were fully vaccinated and 2,088 (1.8\%) were partly vaccinated. Of the contacts, $5,397(2.1 \%)$ were fully vaccinated and 4,411 were partly vaccinated (1.7\%). Characteristics of indexes and contacts are shown in Table 1 . We calculated the VET via the secondary attack rate (SAR) among close contacts of confirmed index cases: 1 - $\left(\mathrm{SAR}_{\text {vaccinated index }} /\right.$ $\left.\mathrm{SAR}_{\text {unvaccinated index }}\right) \times 100 \%$ [3]. The VE among contacts was calculated as: $1-\left(A R_{\text {vaccinated contacts }} / A R_{\text {unvaccinated conta }}\right.$ 


\section{FIGURE}

Crude attack rate of SARS-CoV-2 among contacts, by vaccination status of the index (left to right) and vaccination status of the contact (top to bottom), the Netherlands, 1 February-27 May 2021 ( $\mathrm{n}=113,582$ index cases, $\mathrm{n}=253,168$ contacts)

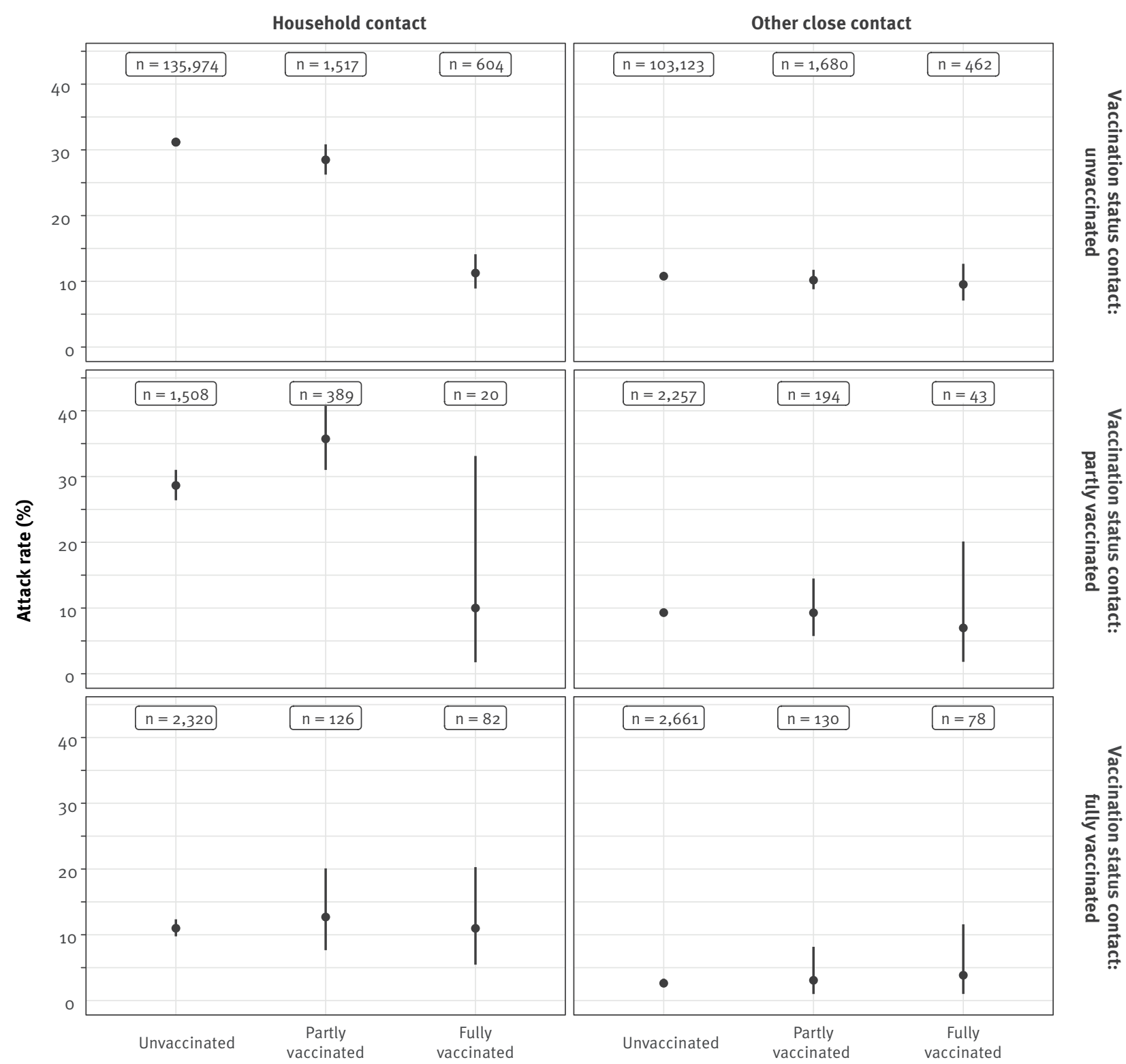

Vaccination status index

SARS-CoV-2: severe acute respiratory syndrome coronavirus 2.

The $\mathrm{n}$ subtotals in the panels indicate the number of unvaccinated/partly vaccinated/fully vaccinated household and other close contacts of unvaccinated/partly vaccinated/fully vaccinated index cases. For example, $n=604$ in the upper left panel denotes that fully vaccinated index cases had 604 unvaccinated household contacts. 


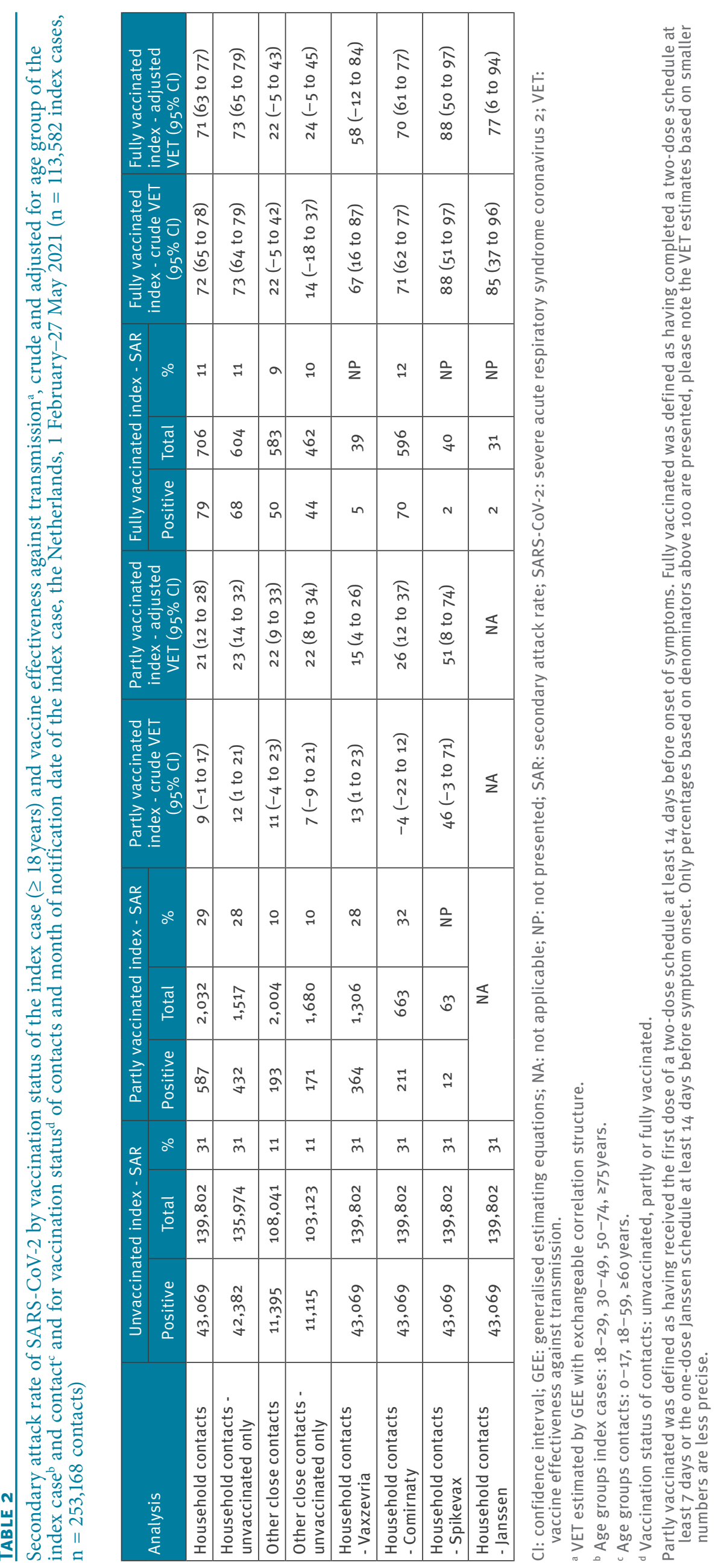




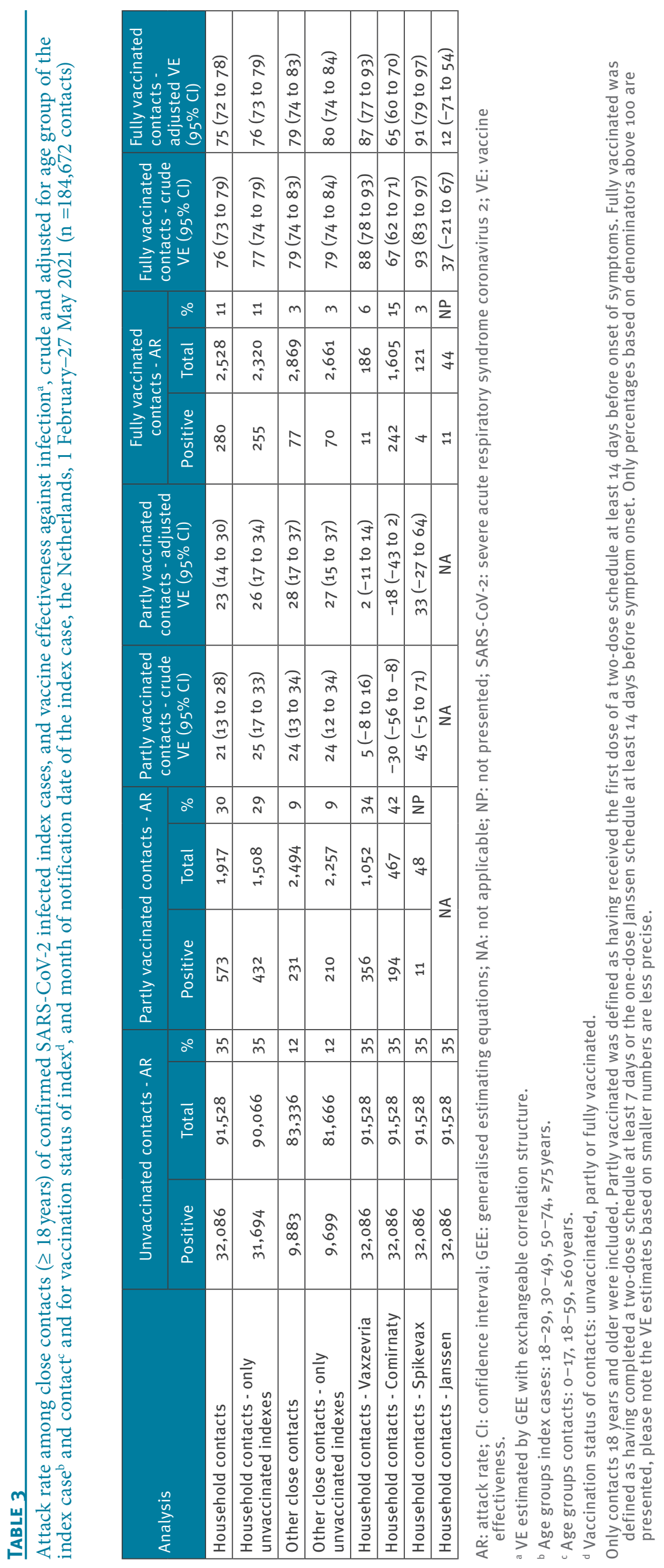


) $\times 100 \%$. Both VET and VE were estimated using a binomial generalised linear model. For parameter fitting we used the generalised estimating equations approach with exchangeable correlation structure to account for clustering of contacts belonging to the same index case [4].

\section{Vaccine effectiveness against transmission}

The Figure shows the crude attack rates among contacts, in relation to the vaccination status of both index and contact. The SAR was 31\% among household contacts of unvaccinated index cases and $11 \%$ among household contacts of fully vaccinated index cases (Table 2). Adjusting for age of the index and contact, vaccination status of the contact and month of notification date of the index case, the VET to household contacts after full vaccination was $71 \%$ (95\% confidence interval $(\mathrm{Cl}): 63$ to 77$)$. The VET to other close contacts was much lower (22\%; $95 \% \mathrm{Cl}:-5$ to 43 ), probably because of the larger risk of the contact being infected through another source (i.e. misclassification of the index case). Stratified by vaccine received by the index case, VET values were estimated at $58 \%$ for Vaxzevria (ChAdOx1-S; AstraZeneca, Cambridge, United Kingdom), 70\% for Comirnaty (BNT162b2; BioNTech/Pfizer, Mainz, Germany/New York, United States (US)), 88\% for Spikevax (mRNA-1273, Moderna, Cambridge, US) and $77 \%$ for the Janssen vaccine. For all vaccines with a two-dose schedule, the adjusted VET (aVET) after one dose was considerably lower than after two doses: $15 \%$ for Vaxzevria, $26 \%$ for Comirnaty and $51 \%$ for Spikevax (Table 2 ).

\section{Vaccine effectiveness among contacts}

The adjusted VE (aVE) for fully vaccinated household contacts of confirmed cases was estimated at $75 \%$ ( $95 \% \mathrm{Cl}: 72$ to 78 ) and for fully vaccinated other close contacts at $79 \%$ (95\% Cl: 74 to 83 ) (Table 3). Stratified by the vaccine received by the contact, aVE was $87 \%$ for Vaxzevria, 65\% for Comirnaty, $91 \%$ for Spikevax and $12 \%$ for Janssen's vaccine. Note that the estimate for the Janssen vaccine was based on only 44 vaccinated contacts, with a median time since vaccination of 21 days. The proportion of vaccinated contacts with unknown vaccine manufacturer was large (Table 1), which reduces the power of the analyses stratified by vaccine.

\section{Discussion}

We estimated a VET of $71 \%$ among household contacts of fully vaccinated index cases. Harris et al. found a VET of $40-50 \%$ for unvaccinated households contacts [5]. That study mostly included partly vaccinated index cases. A study among household contacts of healthcare workers by Shah et al. found a 30\% (after the first dose) and 64\% (after the second dose) reduction in the risk of confirmed SARS-CoV-2 infection among household members of vaccinated healthcare workers, however these healthcare workers were not confirmed as index cases [6].
Martínez-Baz et al. recently estimated VE among 20,961 close contacts of confirmed cases in Spain [7]. In that study, the VE for two doses of Comirnaty was $65 \%(95 \% \mathrm{Cl}: 56$ to 73 ) against infection, which is in agreement with our finding of $65 \%$ VE for this vaccine. These estimates are lower than VE from other observational post-marketing studies. Possibly, the VE against infection is lower when there is high and prolonged exposure to SARS-CoV-2, which is likely for household contacts of confirmed cases [8]. Of note, Martínez-Baz et al. showed an aVE for Comirnaty of $94 \%$ (95\% Cl: 60 to 99) against hospitalisation among close contacts of confirmed cases.

As our study used data not primarily collected for research purposes, it has some important limitations. Our data do not contain information on negative tests among contacts, therefore we do not know if contacts did not get infected or did not seek testing. However, it is likely that close contacts were tested regardless of vaccination status, as the quarantine period for close contacts at the time was reduced from 10 to 5 days when tested negative on day 5 after exposure. For contacts who tested positive, data on vaccination status were more complete because missing data could be supplemented from the notifications. We explored whether this differential completeness influenced our results by excluding all index cases with any household contact with unknown vaccination status or date of vaccination, and the results were the same (data not shown).

Although we tried to minimise misclassification of indexes and contacts by excluding index cases infected at home and contacts with symptom onset before or at the same time as the index, it is plausible that in some instances, the transmission route was reversed or transmission occurred though another source (especially for non-household contacts). If some contacts of vaccinated index cases were infected through other sources, our VET is an underestimation. In addition, we do not have reliable data on the symptoms of the included index cases. Because our analysis on household contacts was restricted to notified index cases not infected at home, probably most of these index cases sought testing because they had symptoms. Symptomatic cases may have been misclassified as index cases in a household, where in reality an asymptomatic household member was the source of transmission to the supposed index case and a third household member. If vaccinees are more likely to be asymptomatic, this source of misclassification may result in an overestimation of the VET.

As the Alpha variant of SARS-CoV-2 dominated during the study period, an important question is to what extent these VET and VE estimates hold in the context of the Delta variant (Pango lineage designation B.1.617.2) which is now dominant in the Netherlands. Also, further research is needed to determine whether the observed differences between the different 
vaccines are due to the small sample size or have real public health relevance. We will prospectively monitor both VET and VE among household contacts over the next months to address these questions.

\section{Conclusion}

Our study showed that the COVID-19 vaccines not only protect the vaccinee against SARS-CoV-2 infection, but also offer protection against transmission to close contacts after completing the full schedule. This finding underscores the importance of full vaccination of close contacts of vulnerable persons.

Members of the RIVM COVID-19 surveillance and epidemiology team:

Agnetha Hofhuis, Anne Teirlinck, Alies van Lier, Bronke Boudewijns, Carolien Verstraten, Guido Willekens, Irene Veldhuijzen, Jan Polman, Jan van de Kassteele, Jeroen Alblas, Janneke van Heereveld, Janneke Heijne, Kirsten Bulsink, Lieke Wielders, Liselotte van Asten, Liz Jenniskens, Loes Soetens, Maarten Mulder, Maarten Schipper, Marit de Lange, Naomi Smorenburg, Nienke Neppelenbroek, Patrick van den Berg, Priscila de Oliveira Bressane Lima, Rolina van Gaalen, Sara Wijburg, Senna van lersel, Siméon de Bruijn, Stijn Andeweg, Sjoerd Wierenga, Susan Lanooij, Sylvia Keijser, Tara Smit, Don Klinkenberg, Jantien Backer, Pieter de Boer, Scott McDonald, Amber Maxwell, Annabel Niessen, Brechje de Gier, Danytza Berry, Daphne van Wees, Dimphey van Meijeren, Eric R.A. Vos, Frederika Dijkstra, Jeanet Kemmeren, Kylie Ainslie, Marit Middeldorp, Marjolein Kooijman, Mirjam Knol, Shahabeh Abbas Zadeh, Timor Faber, Albert Jan van Hoek, Eveline Geubbels, Hester de Melker, Jacco Wallinga, Rianne van Gageldonk-Lafeber, Susan Hahne, Susan van den Hof

\section{Acknowledgements}

The authors would like to thank all source and contact tracing personnel at the 25 Municipal Health Services (GGDen) who have been invaluable for control and surveillance of the COVID-19 epidemic in the Netherlands, and who collected these important data. Funding: This work was funded by the Ministry of Health, Welfare and Sports.

\section{Conflict of interest}

None declared.

\section{Authors' contributions}

Study design: Brechje de Gier, Stijn Andeweg, Jan van de Kassteele, Mirjam Knol. Data collection, management and quality control: Rosa Joosten, Ronald ter Schegget, Stijn Andeweg, Naomi Smorenburg, the RIVM COVID-19 surveillance and epidemiology team. Data analysis: Brechje de Gier, Stijn Andeweg. Interpretation of the data: Brechje de Gier, Stijn Andeweg, Susan Hahné, Susan van den Hof, Hester de Melker, Mirjam Knol. Manuscript draft: Brechje de Gier. All authors critically revised the manuscript and approved the final manuscript.
1. de Gier B, de Oliveira Bressane Lima P, van Gaalen RD, de Boer PT, Alblas J, Ruijten M, et al. Occupation- and age-associated risk of SARS-CoV-2 test positivity, the Netherlands, June to October 2020. Euro Surveill. 2020;25(50):2001884. https:// doi.org/10.2807/1560-7917.ES.2020.25.50.2001884 PMID: 33334396

2. Center for Infectious Disease Control. Protocol bron- en contactonderzoek COVID-19. [Protocol source and contact tracing]. Bilthoven: Rijksinstituut voor Volksgezondheid en Milieu; 8 July 2021. Dutch. Available from: https://lci.rivm.nl/ COVID-19-bco

3. Snijders BEP, van Lier A, van de Kassteele J, Fanoy EB, Ruijs WLM, Hulshof F, et al. Mumps vaccine effectiveness in primary schools and households, the Netherlands, 2008. Vaccine. 2012;30(19):2999-3002. https://doi.org/10.1016/j. vaccine.2012.02.035 PMID: 22381073

4. Halloran ME, Préziosi M-P, Chu H. Estimating vaccine efficacy from secondary attack rates. J Am Stat Assoc. 2003;98(461):3846. https://doi.org/10.1198/016214503388619076

5. Harris RJ, Hall JA, Zaidi A, Andrews NJ, Dunbar JK, Dabrera G. Impact of vaccination on household transmission of SARS-COV-2 in England. London: Knowledge Hub; 2021. Available from: https://khub.net/ documents/135939561/390853656/Impact+of+vaccination+ on+household+transmission+of+SARS-COV-2+in+England. pdf/35bf4bb1-6ade-d3eb-a39e-9c9b25a8122a?t=1619551571214

6. Shah AS, Gribben C, Bishop J, Hanlon P, Caldwell D, Wood $R$, et al. Effect of vaccination on transmission of COVID-19: an observational study in healthcare workers and their households. medRxiv. 2021:2021.03.11.21253275.

7. Martínez-Baz I, Miqueleiz A, Casado I, Navascués A, Trobajo-Sanmartín C, Burgui C, et al. Effectiveness of COVID-19 vaccines in preventing SARS-CoV-2 infection and hospitalisation, Navarre, Spain, January to April 2021. Euro Surveill. 2021;26(21):2100438. https://doi.org/10.2807/15607917.ES.2021.26.21.2100438 PMID: 34047271

8. Halloran ME, Haber M, Longini IM Jr, Struchiner CJ. Direct and indirect effects in vaccine efficacy and effectiveness. Am J Epidemiol. 1991;133(4):323-31. https://doi.org/10.1093/ oxfordjournals.aje.a115884 PMID: 1899778

License, supplementary material and copyright

This is an open-access article distributed under the terms of the Creative Commons Attribution (CC BY 4.0) Licence. You may share and adapt the material, but must give appropriate credit to the source, provide a link to the licence and indicate if changes were made.

Any supplementary material referenced in the article can be found in the online version.

This article is copyright of the authors or their affiliated institutions, 2021. 